Linköping studies in science and technology

Licentiate Thesis. No. 1804

\title{
Optimal Braking Patterns and Forces in Autonomous Safety-Critical Maneuvers
}

\author{
Victor Fors
}

\section{I.U UNNGPENG}

Department of Electrical Engineering

Linköping University, SE-581 33 Linköping, Sweden

Linköping 2018 
Linköping studies in science and technology

Licentiate Thesis. No. 1804

This is a Swedish Licentiate's Thesis.

Swedish postgraduate education leads to a Doctor's degree and/or a Licentiate's degree.

A Doctor's degree comprises 240 ECTS credits (4 years of full-time studies).

A Licentiate's degree comprises 120 ECTS credits,

of which at least 60 ECTS credits constitute a Licentiate's thesis.

Victor Fors

victor.fors@liu.se

www.vehicular.isy.liu.se

Division of Vehicular Systems

Department of Electrical Engineering

Linköping University

SE-581 33 Linköping, Sweden

Copyright (c) 2018 Victor Fors, unless otherwise noted.

All rights reserved.

Fors, Victor

Optimal Braking Patterns and Forces in Autonomous Safety-Critical Maneuvers

ISBN 978-91-7685-301-6

ISSN 0280-7971

Typeset with $\mathrm{IAT}_{\mathrm{E}} \mathrm{X}{ }_{\varepsilon}$

Printed by LiU-Tryck, Linköping, Sweden 2018 


\section{ABSTRACT}

The trend of more advanced driver-assistance features and the development toward autonomous vehicles enable new possibilities in the area of active safety. With more information available in the vehicle about the surrounding traffic and the road ahead, there is the possibility of improved active-safety systems that make use of this information for stability control in safety-critical maneuvers. Such a system could adaptively make a trade-off between controlling the longitudinal, lateral, and rotational dynamics of the vehicle in such a way that the risk of collision is minimized. To support this development, the main aim of this licentiate thesis is to provide new insights into the optimal behavior for autonomous vehicles in safety-critical situations. The knowledge gained have the potential to be used in future vehicle control systems, which can perform maneuvers at-the-limit of vehicle capabilities.

Stability control of a vehicle in autonomous safety-critical at-the-limit maneuvers is analyzed by the use of optimal control. Since analytical solutions of the studied optimal control problems are intractable, they are discretized and solved numerically. A formulation of an optimization criterion depending on a single interpolation parameter is introduced, which results in a continuous family of optimal coordinated steering and braking patterns. This formulation provides several new insights into the relation between different braking patterns for vehicles in at-the-limit maneuvers. The braking patterns bridge the gap between optimal lane-keeping control and optimal yaw control, and have the potential to be used for future active-safety systems that can adapt the level of braking to the situation at hand. A new illustration named attainable force volumes is introduced, which effectively shows how the trajectory of a vehicle maneuver relates to the attainable forces over the duration of the maneuver. It is shown that the optimal behavior develops on the boundary surface of the attainable force volume. Applied to lane-keeping control, this indicates a set of control principles similar to those analytically obtained for friction-limited particle models in earlier research, but is shown to result in vehicle behavior close to the globally optimal solution also for more complex models and scenarios. 



\section{ACKNOWLEDGMENTS}

At all my places of employment I have been lucky to have great coworkers, the Vehicular Systems group at Linköping University where this research has been carried out is no exception. I would like to thank my past and present coworkers for a supportive work environment and productive coffee breaks. I would specifically like to thank my co-authors Lars Nielsen and Björn Olofsson for taking the time to be involved in our papers. Additionally, I would like to thank them and Jan Åslund for their roles as supervisor and co-supervisors.

Other mentions go to Maria Hamnér and Vaheed Nezhadali for showing me the way around the office, Python for providing me with pastries on credit, Kristoffer Lundahl and Pavel Anistratov for being office mates that I can disturb, and any other who influenced the quality of this thesis.

Linköping, May 2018

Victor Fors

\section{FUNDING}

The author is a member of the ELLIIT Excellence Center, the Strategic Area for ICT research, supported by the Swedish Government. This work was partially supported by the Wallenberg AI, Autonomous Systems and Software Program (WASP) funded by the Knut and Alice Wallenberg Foundation. 

Contents

1 Introduction 1

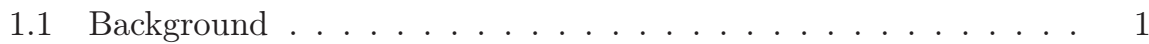

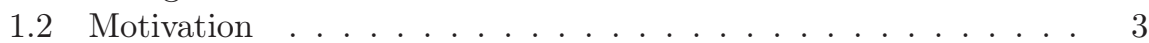

1.3 Contributions ........................ 4

2 Vehicle Model

2.1 World Model . . . . . . . . . . . . . . . . . . . . 9

2.2 Chassis Model . . . . . . . . . . . . . . . . . . 9

2.3 Wheel and Tire Model . . . . . . . . . . . . . . . . . . . 12

$\begin{array}{lr}\text { References } & 17\end{array}$

$\begin{array}{ll}\text { Papers } & 21\end{array}$

I A Continuous Family of Optimal Braking Patterns in Autonomous Safety-Critical Maneuvers 23

1 Introduction . . . . . . . . . . . . . . . . . . . . . . . 24

1.1 Background ................... 24

1.2 Contributions - A Family of Braking Patterns . . . . . . 25

2 Modeling .................... 25

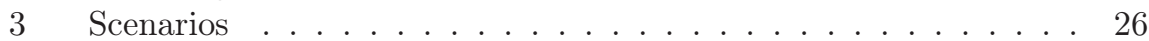

3.1 Left-Hand Turn Scenario . . . . . . . . . . . . . . 26

3.2 Double Lane-Change Scenario . . . . . . . . . . . . . . . . . 27

3.3 Avoidance-Maneuver Scenario . . . . . . . . . . . . 28

4 Optimal Control Problem Formulation . . . . . . . . . . . . . . . 29

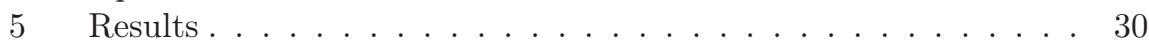

5.1 Solution Method for the OCP . . . . . . . . . . 30

5.2 Additional Interpretation and Evaluation Measures . . . . 30

5.3 Left-Hand Turn . . . . . . . . . . . . . . . . . . . . . . . . 31

5.4 Double Lane-Change . . . . . . . . . . . . . . . . . . . 36

5.5 Avoidance Maneuver . . . . . . . . . . . . . . . . 36 
6 Discussion . . . . . . . . . . . . . . . . . . . . . 39

6.1 General Observations Regarding Braking Patterns . . . . 39

6.2 Different Road Conditions . . . . . . . . . . . . . . 41

6.3 Braking Patterns for Lane-Keeping Control Design . . . . 41

7 Conclusions . . . . . . . . . . . . . . . . . 42

References....................... . . . 42

II Attainable Force Volumes of Optimal

Autonomous at-the-limit Maneuvers $\quad 45$

1 Introduction . . . . . . . . . . . . . . . . . . . 46

1.1 Background . . . . . . . . . . . . . 46

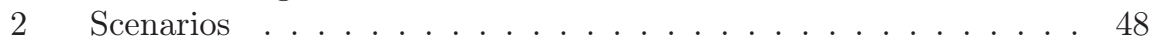

2.1 Left-Hand Turn Scenario . . . . . . . . . . . . . . . . 48

2.2 Double Lane-Change Scenario . . . . . . . . . . . . . . . . 48

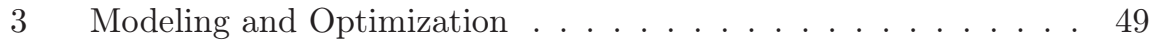

3.1 Vehicle Model . . . . . . . . . . . . . . . 50

3.2 Optimal Control Problem . . . . . . . . . . . . 51

4 Forces ........................ 51

4.1 Global Forces . . . . . . . . . . . . . . . . 52

4.2 Attainable Forces From Individual Braking . . . . . . . . 57

4.3 Attainable Force Volumes . . . . . . . . . . . . . . . . 58

5 Control Forces During the Left-Hand Turn . . . . . . . . . . . . . 59

6 Attainable Forces During the Left-Hand Turn . . . . . . . . . . . 60

7 Attainable Force Volumes During Critical Maneuvers . . . . . . . 60

7.1 Left-Hand Turn . . . . . . . . . . . . . . . 61

7.2 Double Lane-Change . . . . . . . . . . . . . . . . . . . 65

8 Global Forces for Controller Design . . . . . . . . . . . . . . . 67

9 Conclusions . . . . . . . . . . . . . . . . . 68

References........................ 69 


\section{Chapter 1}

\section{Introduction}

The modern passenger car is not only defined by its hardware, but also the millions of lines of code used to control and monitor its different systems and present information to the driver. Cars are traditionally controlled by human drivers, who has to rely on their own senses and driving knowledge to handle any situation that may arise. As the amount of sensors in and on cars increase, the computational hardware improves, and new algorithms are developed, autonomous vehicle control systems can start to outperform even the driving performance of professional drivers. The main aim of this licentiate thesis is to provide new insights into the optimal behavior for autonomous vehicles in safety-critical situations. The knowledge gained have the potential to be used in future vehicle control systems, which can perform maneuvers at-the-limit of vehicle capabilities.

\subsection{BACKGROUND}

Since the mid-20th century the number of deaths because of automotive accidents in Sweden has decreased in number even though the number of vehicles on the road has increased dramatically. In the year 1950, automotive accidents in Sweden resulted in 172.5 deaths per 100,000 cars, the year 2016 that number was down to 5 deaths per 100,000 cars (Transportstyrelsen, 2017). Improvements in the interior design of a vehicle from a safety standpoint can drastically increase the chance of survival in the case of an automotive accident (Shelden, 1955). These safety improvements are referred to as passive safety systems, which include safety features such as seat belts, air bags, and deformation zones. Much of the decrease in the number of deaths because of automotive accidents is thanks to the development and improvement of passive safety systems. However, the number of injured in automotive accidents do not show as steep a decrease 
(Transportstyrelsen, 2017). Passive safety systems do not prevent accidents from happening, but significantly reduce the risk of serious injury or death.

Driver-assistance systems that can reduce the risk of accidents are referred to as active-safety systems. A commonly cited example is the anti-lock braking system (ABS), which prevents the loss of steering ability during heavy braking and can decrease the stopping distance (Kiencke and Nielsen, 2005). Fitting a motorcycle with ABS reduces the risk of accidents and the risk of an accident being severe or fatal (Rizzi et al., 2015). For cars, it is harder to prove that ABS reduces the risk of driver injury (Cummings and Grossman, 2007), specifically ABS is not significantly reducing the risk of run-off-road crashes (Kahane and Dang, 2009). Thankfully, modern cars are also equipped with Electronic Stability Control (ESC), which reduces the risk of run-off-road crashes (Lyckegaard et al., 2015). An ESC system uses differential braking to keep the vehicle response to steering input predictable for the driver (Genta and Morello, 2008), which is especially helpful in scenarios where the desired vehicle behavior is close to or beyond the capabilities of the vehicle.

Classic active-safety systems such as ABS and ESC rely on the driver to take in and process information about the surrounding vehicles and the road ahead, and provide appropriate input in terms of braking and steering. A modern driver-assistance system available in many cars that acts based on information about the surrounding vehicles is adaptive cruise control (ACC) (Rajamani, 2012). ACC is an advancement of the classic cruise control systems that apart from keeping the vehicle driving at a reference velocity, also keeps the distance to the vehicle in front. ACC does still rely on being able to fall back on driver control and is not directly designed to prevent collisions. A corresponding function specifically designed for accident prevention is autonomous emergency braking (AEB), which applies heavy braking without any input from the driver to avoid collisions (Gordon and Lidberg, 2015). Cars with AEB have reduced risk for front-to-rear crashes and many injuries are estimated to be prevented as the function gains more widespread use (Cicchino, 2017).

Indicative of the potential capabilities of future driver-assistance systems, Kapania and Gerdes (2015) and $\mathrm{Ni}$ and $\mathrm{Hu}$ (2017) demonstrate autonomous racing cars driving at-the-limit of handling. It is interesting to consider how the advancement of driver-assistance systems in cars will progress. Gordon and Lidberg (2015) present a view on the development of driver-assistance systems as an evolution of enabling technologies, where the systems build on each other as they gain more and more control authority along with the hardware required to support them. One of the major driving forces behind the development of advanced driver-assistance systems and active safety is the promising prospect of fully autonomous vehicles. In an automated driving system as defined by the SAE International (2016) and adopted by the NHTSA (2016), the human driver is not required to monitor the driving environment and is at most only conditionally required to intervene. This definition means that these system can not rely on falling back on the driver when an unexpected safety-critical 


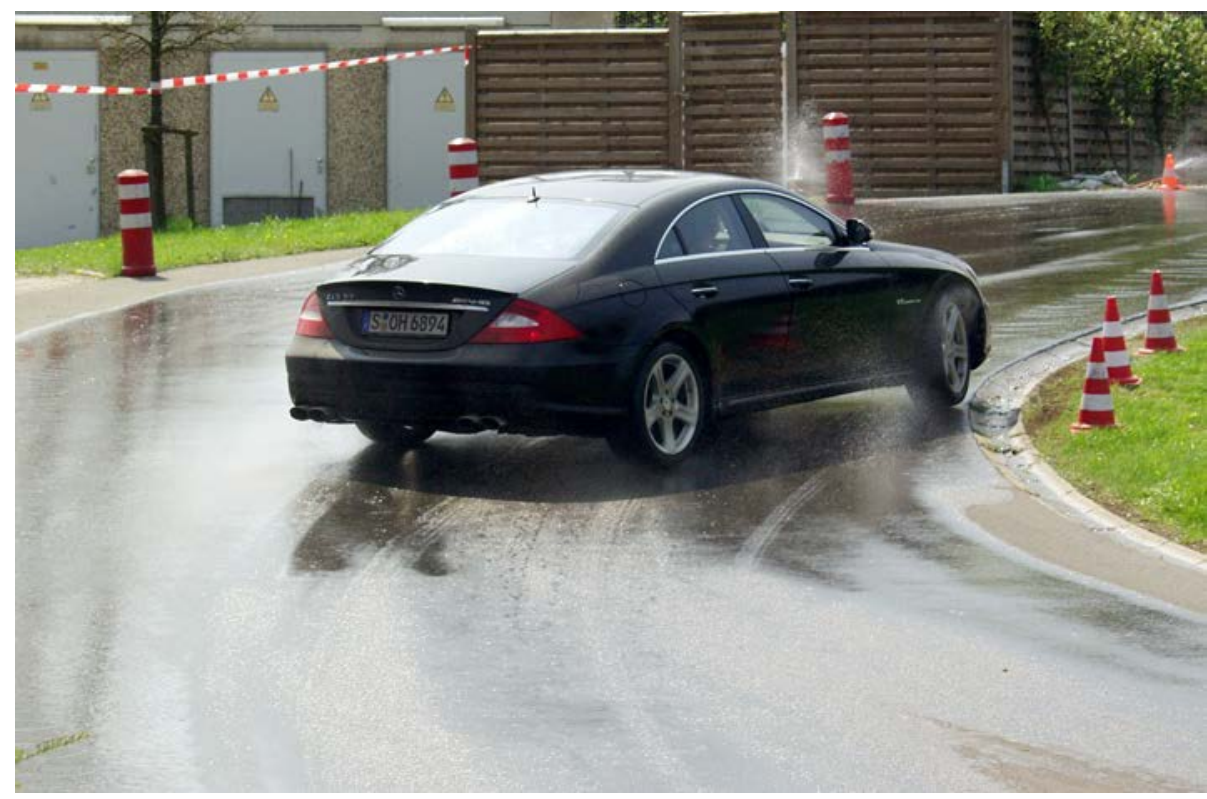

Figure 1.1: Car sliding on wet asphalt. Source: (c) Raimond Spekking / CC BY-SA 4.0 (via Wikimedia Commons)

situation occurs, putting high requirements on their robustness, and creating a demand for autonomous solutions to critical situations. Semi-autonomous features that not only control the acceleration and braking of the vehicle, but also control the steering, are already today available in commercial passenger cars. Pilot projects for autonomous vehicles driving on public road are taking place in various locations around the world (Muoio, 2017).

\subsection{Motivation}

The increased situational awareness required for semi-autonomous or fully autonomous driving is enabling a new generation of autonomous active-safety systems. At the same time, active safety purposed for autonomous driving must be able to handle a wide range of scenarios for an automated driving system to be as safe as a human driver in all situations. Starting with AEB, which given information about objects in front can initiate heavy braking without any driver command, there is the possibility to expand the capabilities of these systems to handle the more general task of collision avoidance by both braking and steering. Braking in a situation where only longitudinal dynamics has to be considered is relatively straightforward, where close to optimal performance can be achieved by relying on the ABS to keep the tire forces close to the limit of friction. When 
also lateral dynamics has a significant impact on the task of collision avoidance, perhaps requiring vehicle maneuvers similar to what is depicted in Figure 1.1, the problem becomes more complex. At a high-level, the system needs to adaptively make a trade-off between controlling the longitudinal, lateral, and rotational dynamics of the vehicle in such a way that the risk of collision is minimized. Optimal control has many applications related to vehicle dynamics (Olofsson, 2015, Sharp and Peng, 2011, Waschl et al., 2014), and can in this case be used to find control principles to support the development of future active-safety systems capable of handling situations at-the-limit of vehicle performance. Such control principles should be valid for vehicle models sufficiently complex to capture the essential dynamics of a real car, while still remaining valid for a range of vehicles and scenarios.

\subsection{COnTRibutions}

The main contributions are summarized below for each paper included in this thesis.

\section{Paper I: A Continuous Family of Optimal Braking Patterns in Autonomous Safety-Critical Maneuvers}

In this paper, we investigate optimal braking patterns in completely autonomous safety-critical maneuvers in the relevant scenarios of optimal braking and steering in a left-hand turn, in a double lane-change, and in an avoidance situation. A formulation of an optimization criterion depending on a single interpolation parameter is introduced, which results in a continuous family of braking patterns. This formulation provides several new insights into the relation between different braking patterns for vehicles in at-the-limit maneuvers. The braking patterns show significant similarities in behavior for the different scenarios and bridge the gap between optimal lane-keeping control and optimal yaw control. This points forward toward future lane-keeping control systems that can seamlessly adapt the level of braking depending on the situation at hand.

\section{Paper II: Attainable Force Volumes of Optimal Autonomous AT-The-Limit Maneuvers}

In this paper, the attainable forces of optimal autonomous maneuvers are examined and compared to the actual force utilization in optimal vehicle maneuvers. A new illustration named attainable force volumes is introduced, and it is used to effectively show how the trajectory of a vehicle maneuver relates to the attainable forces over the duration of the maneuver. The optimal trajectories are shown to typically be on the boundary surface of the attainable force volume. Applied to lane-keeping control, this indicates a set of control principles similar to those analytically obtained for friction-limited particle models in earlier research, but 
is shown to result in vehicle behavior close to the globally optimal solution also for more complex models and scenarios.

\section{Other Publications by the Author}

- Victor Fors, Björn Olofsson, and Lars Nielsen. Formulation and Interpretation of Optimal Braking Patterns in Autonomous Lane-Keeping Maneuvers. 2nd IAVSD Workshop on Dynamics of Road Vehicles. Berlin, Germany, 2017.

- Victor Fors, Björn Olofsson, and Lars Nielsen. Slip-Angle Feedback Control for Autonomous Safety-Critical Maneuvers at-the-limit of Friction. 14th International Symposium on Advanced Vehicle Control. Beijing, China, 2018. Accepted for oral presentation. 



\section{Chapter 2}

\section{Vehicle Model}

The characteristics of optimal maneuvers can be highly dependent on the road condition and the vehicle model used, see (Olofsson et al., 2013) and (Berntorp et al., 2014), respectively. It is therefore important that the vehicle model used is able to handle important phenomena such as load transfer. Meanwhile, it is also desirable for the model to not be overly specified, in order for the result to not be more dependent on specific vehicle parameters than necessary and remain representative for the general behavior of a wider range of vehicles. The impact the specific vehicle model parameters has on robustness of the achieved driving behavior is examined by comparing the behavior for different scenarios and parameters. The model also needs to be of reasonable computational complexity for the application considered.

For the application of numerical optimal control, this thesis makes extensive use of the vehicle model referred to as DT WF by Berntorp et al. (2014). The model is presented in this chapter for the sake of completeness, providing the reader with an overview of the model equations. The name of the vehicle model used, DT WF, comes from that it is a double-track model using weighting functions (Pacejka, 2006) to model combined longitudinal and lateral tire forces. A double-track model includes wheels on both sides of the vehicle in the model, as illustrated in Figure 2.1. The alternative is to simplify the model to a single-track model by combining the forces produced by the left-hand side and right-hand side wheels into a single centered track with only two wheels. Often, the singletrack vehicle model is able to accurately capture the behavior of the vehicle (Lundahl et al., 2013), but it lacks the ability to model differential braking. An alternative to the weighting functions used to model combined longitudinal and lateral tire forces (see Section 2.3), is the commonly used friction-ellipse based model (Wong, 2008). In the friction-ellipse based model the longitudinal tire force is the input and no additional parameters are needed compared to when calculating the nominal tire forces. A potential problem with the friction-ellipse 
Table 2.1: Notation for vehicle model states.

\begin{tabular}{ll}
\hline Description & Notation \\
\hline Position coordinates & $X_{p}, Y_{p}$ \\
Orientation & $\psi$ \\
Longitudinal velocity & $v_{x}$ \\
Lateral velocity & $v_{y}$ \\
Yaw rate & $r$ \\
Roll angle and roll rate & $\phi, \dot{\phi}$ \\
Pitch angle and pitch rate & $\theta, \dot{\theta}$ \\
Steering angle & $\delta$ \\
Rotational wheel speeds & $\omega_{1}, \omega_{2}, \omega_{3}, \omega_{4}$ \\
Wheel slip angles & $\alpha_{1}, \alpha_{2}, \alpha_{3}, \alpha_{4}$ \\
Applied wheel torques & $T_{1}, T_{2}, T_{3}, T_{4}$ \\
\hline
\end{tabular}

Table 2.2: Notation for vehicle model inputs.

\begin{tabular}{ll}
\hline Description & Notation \\
\hline Steering rate & $\dot{\delta}$ \\
Commanded wheel torques & $T_{u, 1}, T_{u, 2}, T_{u, 3}, T_{u, 4}$ \\
\hline
\end{tabular}

based model is that it does not model any limitation on the longitudinal tire force as a function of the potential lateral tire force. At the extreme, a tire experiencing maximum lateral tire force could be braked to produce maximum longitudinal tire force, which is obviously an unrealistic behavior. In contrast, for the weighting function model adopted in Section 2.3, the longitudinal and lateral tire forces are implicitly dependent on each other by the states of the wheel and the tire, which is much more in line with experimental data found in, e.g., (Pacejka, 2006).

The vehicle model has in total 23 states distributed as follows: 3 for the global vehicle position and orientation, 3 for the vehicle velocity and yaw rate, 4 for the motion of the suspended vehicle body, 1 for the steering angle, and 3 for each of the four wheels for wheel and tire dynamics. The model has 5 inputs: the steering rate and the commanded torque at each of the four wheels. The states and inputs of the vehicle model are listed in Table 2.1 and Table 2.2, respectively. Their relations are described in the following sections:

- Section 2.1 describes the vehicle states in the world coordinate system.

- Section 2.2 describes the chassis and suspension system.

- Section 2.3 describes the individual wheel and road-tire dynamics. 

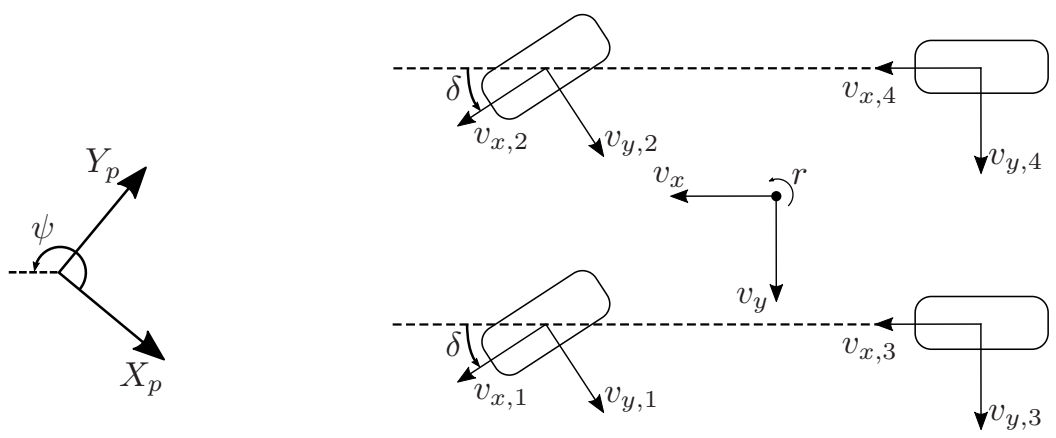

Figure 2.1: Double-track vehicle model and its associated global coordinate system.

\subsection{WORLD MOdEL}

The vehicle traverses in a global coordinate system, where its position $\left(X_{p}, Y_{p}\right)$ and orientation $\psi$ are defined by the differential relations

$$
\begin{aligned}
\dot{\psi} & =r, \\
\dot{X}_{p} & =v_{x} \cos (\psi)-v_{y} \sin (\psi), \\
\dot{Y}_{p} & =v_{x} \sin (\psi)+v_{y} \cos (\psi),
\end{aligned}
$$

where $r$ is the yaw rate, $v_{x}$ is the longitudinal velocity, and $v_{y}$ is the lateral velocity, as defined in Figure 2.1. By using the state variables $X_{p}, Y_{p}$, and $\psi$, the interaction of the vehicle with the world is defined by putting constraints on these states of the form $f\left(X_{p}, Y_{p}, \psi\right) \leq 0$, e.g., to model the space outside lane borders or inside obstacles that the vehicle can not occupy.

\subsection{Chassis Model}

The global position, $\left(X_{p}, Y_{p}\right)$, marks the center of rotation in the vehicle frame, which is fixed along the ground plane. The vehicle center of mass is located at a fixed distance $h$ from this point, with its position dependent on both pitch and roll dynamics. The pitch angle $\theta$ and the roll angle $\phi$ are defined in Figure 2.2 as the coordinate transformation from the vehicle frame $(x, y, z)$, to the suspended vehicle body frame $\left(x^{\prime \prime}, y^{\prime \prime}, z^{\prime \prime}\right)$. The position of the vehicle center of mass is constant in the fixed frame of the vehicle body, positioned a distance $h$ along the $z^{\prime \prime}$-axis in Figure 2.2. The suspension is modeled as independent spring-damper systems acting on the rotational degrees of freedom $\theta$ and $\phi$. This modeling means that coupled effects of the suspension system from combined roll and pitch motion are not taken into account, but for the purpose of this thesis it is sufficient to model the characteristics of typical load transfer effects. 


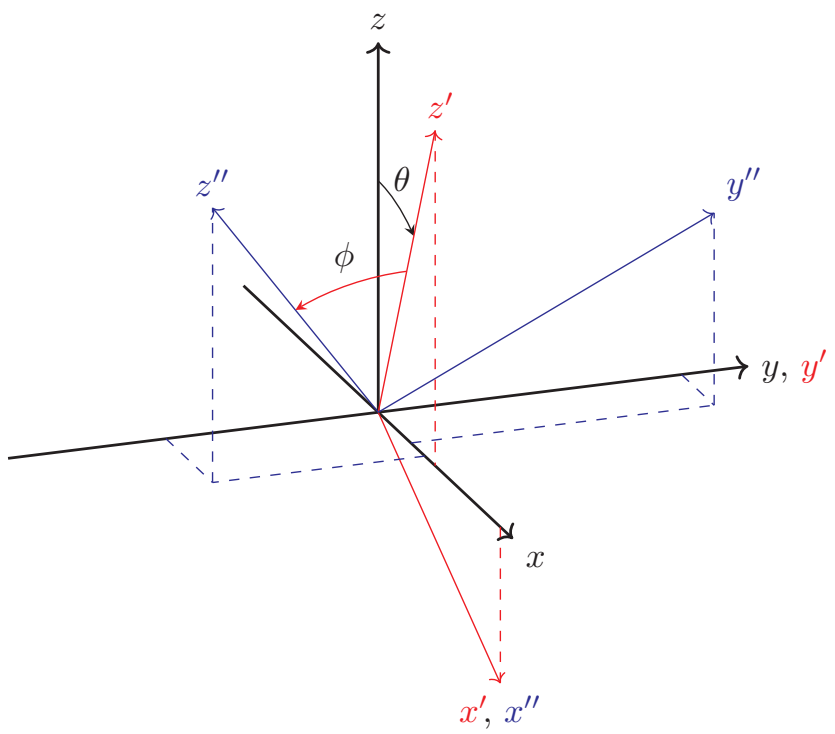

Figure 2.2: Coordinate transform from vehicle coordinates to vehicle-body coordinates. In this figure, $x$ is the longitudinal axis of the vehicle frame, $y$ is the lateral axis of the vehicle frame, and $z$ is the vertical axis of the vehicle frame. The pitch angle $\theta$ is the rotation around the $y$-axis and the roll angle $\phi$ is the rotation around the transformed $x^{\prime}$-axis.

Counted from left to right and front to rear in the vehicle ego orientation, the vehicle has four wheels denoted with subscript $i \in\{1,2,3,4\}$, see Figure 2.1. Each wheel $i$ is fixed along the ground plane, at a longitudinal and lateral distance $\left(l_{x, i}, l_{y, i}\right)$ from the center of rotation in the vehicle frame. On real cars, the steering angle influences the wheel angles of the front wheels differently, typically according to Ackerman steering geometry (Wong, 2008). In this model, the geometry is simplified by assuming that wheels on the same axle have equal wheel angle. The angle of the two wheels on the front axle is defined by the steering angle, $\delta$, according to

$$
\delta_{1}=\delta_{2}=\delta
$$

while the angle of the rear wheels is zero, i.e.,

$$
\delta_{3}=\delta_{4}=0
$$

Each tire influences the chassis dynamics by its interaction with the road, see Section 2.3. The variables $F_{x, i}$ and $F_{y, i}$ denotes the force components from the tire traction of each wheel $i$ in the local coordinate system of the wheel. The total forces and the total moment from the tires in the vehicle frame are given 
by

$$
\begin{aligned}
{\left[\begin{array}{l}
F_{x} \\
F_{y}
\end{array}\right] } & =\sum_{i=1}^{4}\left[\begin{array}{cc}
\cos \left(\delta_{i}\right) & -\sin \left(\delta_{i}\right) \\
\sin \left(\delta_{i}\right) & \cos \left(\delta_{i}\right)
\end{array}\right]\left[\begin{array}{l}
F_{x, i} \\
F_{y, i}
\end{array}\right], \\
M_{z} & =\sum_{i=1}^{4}\left[\begin{array}{ll}
l_{x, i} & -l_{y, i}
\end{array}\right]\left[\begin{array}{cc}
\cos \left(\delta_{i}\right) & -\sin \left(\delta_{i}\right) \\
\sin \left(\delta_{i}\right) & \cos \left(\delta_{i}\right)
\end{array}\right]\left[\begin{array}{l}
F_{x, i} \\
F_{y, i}
\end{array}\right] .
\end{aligned}
$$

A summary of the motion equations of the chassis, derived by Berntorp (2013), is given below. The reader is referred to that reference for further details regarding the derivation of the model. From the moving coordinate systems of the vehicle in Figure 2.1, and the roll and pitch motion illustrated in Figure 2.2, the translational motion equations can be written as

$$
\begin{aligned}
& \dot{v}_{x}-v_{y} r+h f_{x}(r, \dot{r}, \phi, \dot{\phi}, \ddot{\phi}, \theta, \dot{\theta}, \ddot{\theta})=\frac{F_{x}}{m}, \\
& \dot{v}_{y}+v_{x} r+h f_{y}(r, \dot{r}, \phi, \dot{\phi}, \ddot{\phi}, \theta, \dot{\theta}, \ddot{\theta})=\frac{F_{y}}{m},
\end{aligned}
$$

where $m$ is the vehicle mass, and $h f_{x}(\cdot)$ and $h f_{y}(\cdot)$ are the contributions from the roll and pitch dynamics. The expressions for $f_{x}$ and $f_{y}$ are stated in (Berntorp, 2014). The rotational dynamic equation for the yaw rate $r$ is given by

$$
\dot{r} I_{r}\left(\theta, \phi, I_{B}\right)=M_{z}+h \tau_{r}\left(F_{x}, F_{y}, \phi, \theta\right),
$$

where $I_{r}(\cdot)$ is the moment of inertia associated with $r, I_{B}$ is the moment of inertia matrix in the fixed frame of the vehicle body, and $h \tau_{r}(\cdot)$ is the additional external torques from the deflection of the center of mass of the chassis. The equation of the pitch dynamics is given by

$$
\ddot{\theta} I_{\theta}\left(\phi, I_{B}\right)+\nu_{\theta}\left(r, \phi, \dot{\phi}, \theta, \dot{\theta}, I_{B}\right)=-\left(D_{\theta} \dot{\theta}+K_{\theta} \theta\right)+h \tau_{\theta}\left(F_{x}, m g, \phi, \theta\right),
$$

where $I_{\theta}(\cdot)$ is the moment of inertia associated with $\theta, \nu_{\theta}(\cdot)$ is the lower-order inertial terms, $D_{\theta} \dot{\theta}+K_{\theta} \theta$ models a spring-damper system, $h \tau_{\theta}(\cdot)$ is the external torques from the deflection of the center of mass of the chassis, and $g$ is the gravitational acceleration constant. The equation of the roll dynamics is given by

$$
\ddot{\phi} I_{\phi}\left(\phi, \theta, I_{B}\right)+\nu_{\phi}\left(r, \phi, \dot{\phi}, \theta, \dot{\theta}, I_{B}\right)=-\left(D_{\phi} \dot{\phi}+K_{\phi} \phi\right)+h \tau_{\phi}\left(F_{y}, m g, \phi, \theta\right),
$$

where $I_{\phi}(\cdot)$ is the moment of inertia associated with $\phi, \nu_{\phi}(\cdot)$ is the lower-order inertial terms, $D_{\phi} \dot{\phi}+K_{\phi} \phi$ models a spring-damper system, and $h \tau_{\phi}(\cdot)$ is the external torques from the deflection of the center of mass of the chassis.

The normal force on each wheel, $F_{z, i}$, influences the tire traction and can be solved from force and torque equilibria. The vertical force equilibrium is given by

$$
F_{z, 1}+F_{z, 2}+F_{z, 3}+F_{z, 4}=m g \text {. }
$$


The longitudinal load-transfer equilibrium is given by the relation

$$
\sum_{i=1}^{4} l_{x, i} F_{z, i}=K_{\theta} \theta+D_{\theta} \dot{\theta}
$$

Finally, the lateral load-transfer equilibria are governed by

$$
\begin{aligned}
& -\left(l_{y, 1} F_{z, 1}+l_{y, 2} F_{z, 2}\right)=K_{\phi} \phi / 2+D_{\phi} \dot{\phi} / 2, \\
& -\left(l_{y, 3} F_{z, 3}+l_{y, 4} F_{z, 4}\right)=K_{\phi} \phi / 2+D_{\phi} \dot{\phi} / 2 .
\end{aligned}
$$

The numerical model parameters employed in the chassis model are detailed in Table 2.3 found at the end of the chapter. The chassis model parameters are from (Lundahl, 2016) and resemble the dynamics of a standard passenger car.

\subsection{Wheel ANd Tire Model}

The equations given in this section are true for each wheel $i \in\{1,2,3,4\}$. The velocity of each wheel in its local coordinate system, $\left(v_{x, i}, v_{y, i}\right)$, is described by

$$
\left[\begin{array}{l}
v_{x, i} \\
v_{y, i}
\end{array}\right]=\left[\begin{array}{cc}
\cos \left(\delta_{i}\right) & \sin \left(\delta_{i}\right) \\
-\sin \left(\delta_{i}\right) & \cos \left(\delta_{i}\right)
\end{array}\right]\left(\left[\begin{array}{l}
v_{x} \\
v_{y}
\end{array}\right]+r\left[\begin{array}{c}
-l_{y, i} \\
l_{x, i}
\end{array}\right]\right) .
$$

The rotational dynamics of a wheel are illustrated in Figure 2.3(a). By calculating the equilibrium of the moment contributions to the wheel rotation, the dynamics for the wheel states, i.e., the rotational speeds $\omega_{i}$, are given by

$$
I_{w} \dot{\omega}_{i}=T_{i}-R_{w} F_{x, i}
$$

where $T_{i}$ is the applied driving or braking torque on wheel $i, I_{w}$ is the rotational wheel inertia, and $R_{w}$ is the wheel radius. To model the braking system, the braking of each wheel is individually actuated by the commanded torque $T_{u, i}$ through a first-order system

$$
\gamma_{T} \dot{T}_{i}+T_{i}=T_{u, i}
$$

where $\gamma_{T}$ is the time constant of the braking system ${ }^{1}$.

To model the ground-tire interaction, the definitions from Pacejka (2006) are used to describe the longitudinal tire slip and the lateral tire slip. The longitudinal slip ratios $\kappa_{i}$ are defined as

$$
\kappa_{i}=\frac{R_{w} \omega_{i}-v_{x, i}}{v_{x, i}} .
$$

\footnotetext{
${ }^{1}$ In this thesis only braking is considered. Note that if both braking and accelerating maneuvers are to be considered, different time constants are appropriate to reflect the different dynamics.
} 


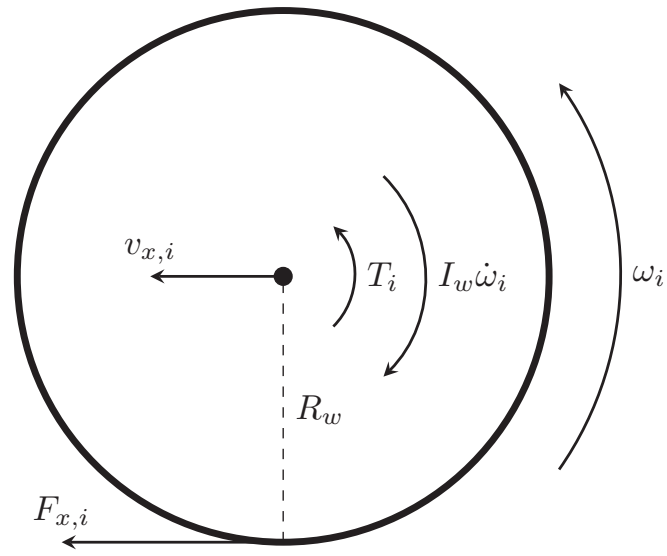

(a) Wheel model seen from the side.

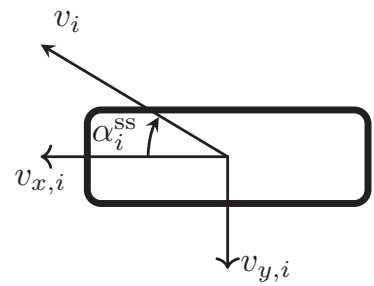

(b) Wheel model seen from above.

Figure 2.3: Wheel model.

With this definition, a locked wheel has the slip ratio $\kappa_{i}=-1$ and a freerolling wheel has the slip ratio $\kappa_{i}=0$. The lateral slip angles $\alpha_{i}$ are, when at their respective steady-state value $\alpha_{i}^{\mathrm{ss}}$, defined as the angle between the wheel ego direction and the velocity vector $v_{i}$ of the wheel, see Figure 2.3(b). The distance traveled by the tire before the slip angle reaches its steady-state value is determined by the relaxation length $\sigma$. The lateral slip angles $\alpha_{i}$ are defined as by Pacejka (2006)

$$
\frac{\sigma}{v_{x, i}} \dot{\alpha}_{i}+\alpha_{i}=\alpha_{i}^{\mathrm{ss}}=-\arctan \left(\frac{v_{y, i}}{v_{x, i}}\right) .
$$

The numerical model parameters employed in the wheel model are detailed in Table 2.4(a) found at the end of the chapter.

The longitudinal forces $F_{x, i}$ and the lateral forces $F_{y, i}$ of the tires are modeled using Pacejka's Magic Formula with weighting functions from Pacejka (2006) and are given by

$$
\begin{aligned}
F_{x 0, i}= & \mu_{x, i} F_{z, i} \sin \left(C _ { x , i } \operatorname { a r c t a n } \left(B_{x, i} \kappa_{i}\right.\right. \\
& \left.-E_{x, i}\left(B_{x, i} \kappa_{i}-\arctan \left(B_{x, i} \kappa_{i}\right)\right)\right), \\
H_{x \alpha, i}= & B_{x 1, i} \cos \left(\arctan \left(B_{x 2, i} \kappa_{i}\right)\right), \\
G_{x \alpha, i}= & \cos \left(C_{x \alpha, i} \arctan \left(H_{x \alpha, i} \alpha_{i}\right)\right), \\
F_{x, i}= & G_{x \alpha, i} F_{x 0, i},
\end{aligned}
$$




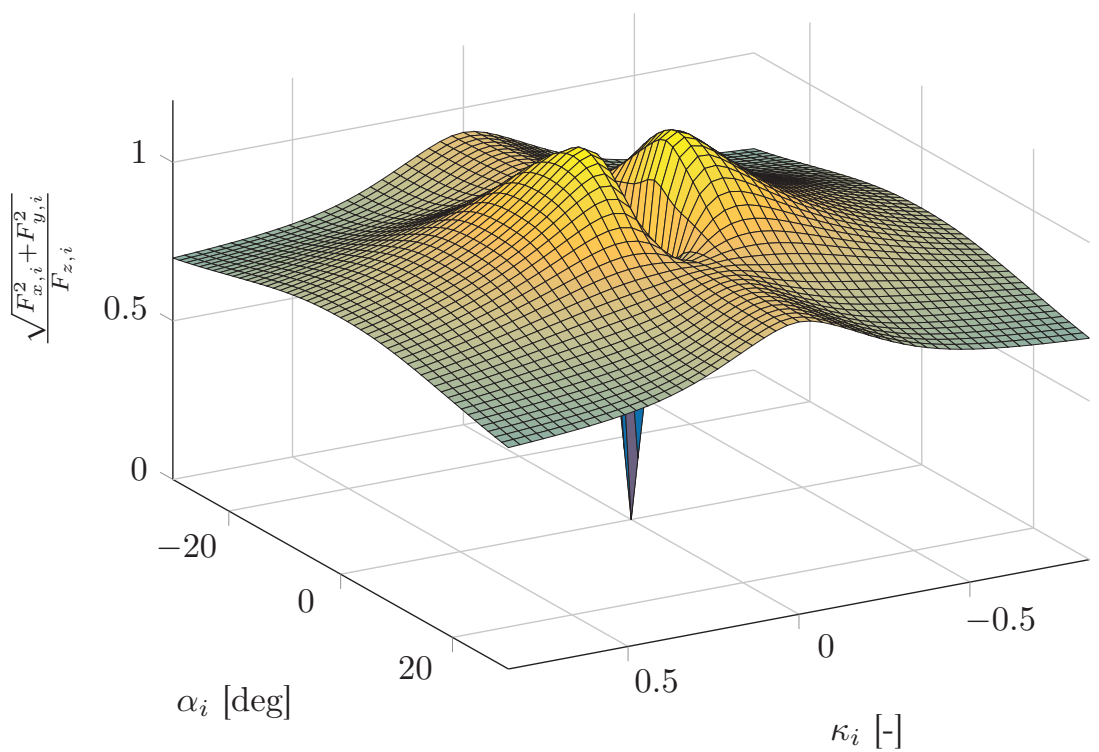

Figure 2.4: Force-slip diagram of Pacejka's Magic Formula with weighting functions using parameters corresponding to dry asphalt interaction. The resultant force is normalized with the normal force.

$$
\begin{aligned}
F_{y 0, i}= & \mu_{y, i} F_{z, i} \sin \left(C _ { y , i } \operatorname { a r c t a n } \left(B_{y, i} \alpha_{i}\right.\right. \\
& \left.-E_{y, i}\left(B_{y, i} \alpha_{i}-\arctan \left(B_{y, i} \alpha_{i}\right)\right)\right), \\
H_{y \kappa, i}= & B_{y 1, i} \cos \left(\arctan \left(B_{y 2, i} \alpha_{i}\right)\right), \\
G_{y \kappa, i}= & \cos \left(C_{y \kappa, i} \arctan \left(H_{y \kappa, i} \kappa_{i}\right)\right), \\
F_{y, i}= & G_{y \kappa, i} F_{y 0, i},
\end{aligned}
$$

where $B, C$ and $E$ are model parameters, and $\mu_{x}$ and $\mu_{y}$ are the friction coefficients. For no lateral slip, $\alpha_{i}$, the resultant force is given by the Magic Formula for the longitudinal force (2.22), and for no longitudinal slip, $\kappa_{i}$, the resultant force is given by the Magic Formula for the lateral force (2.26). An example of the resultant force from different combinations of longitudinal and lateral slip is seen in Figure 2.4. The tire-force model parameters used in the model are collected in Table 2.4. The parameters have been used by Lundahl (2016) and originates from the empirical model presented by Pacejka (2006), representing the behavior of a tire on dry asphalt. 
Table 2.3: Vehicle model parameters for the chassis used in the relations (2.7)(2.17). The chassis model parameters are from (Lundahl, 2016) and resemble the dynamics of a standard passenger car.

\begin{tabular}{|c|c|c|c|}
\hline Description & Notation & Value & Unit \\
\hline \multirow{2}{*}{$\begin{array}{l}\text { Longitudinal distance from the } \\
\text { center of rotation in the vehicle } \\
\text { frame to wheel } i\end{array}$} & $l_{x, 1}, l_{x, 2}$ & 1.3 & $\mathrm{~m}$ \\
\hline & $l_{x, 3}, l_{x, 4}$ & -1.5 & $\mathrm{~m}$ \\
\hline \multirow{2}{*}{$\begin{array}{l}\text { Lateral distance from the center of } \\
\text { rotation in the vehicle frame to } \\
\text { wheel } i\end{array}$} & $l_{y, 1}, l_{y, 3}$ & 0.8 & $\mathrm{~m}$ \\
\hline & $l_{y, 2}, l_{y, 4}$ & -0.8 & $\mathrm{~m}$ \\
\hline Vehicle mass & $m$ & 2100 & $\mathrm{~kg}$ \\
\hline $\begin{array}{l}\text { Height of the mass center in the } \\
\text { fixed frame of the vehicle body }\end{array}$ & $h$ & 0.5 & $\mathrm{~m}$ \\
\hline $\begin{array}{l}\text { Moment of inertia matrix in the } \\
\text { fixed frame of the vehicle body }\end{array}$ & $I_{B}$ & {$\left[\begin{array}{ccc}765 & 0 & 0 \\
0 & 3480 & 0 \\
0 & 0 & 3900\end{array}\right]$} & $\mathrm{kgm}^{2}$ \\
\hline Pitch suspension stiffness & $K_{\theta}$ & 364 & $\mathrm{kNm}(\mathrm{rad})^{-1}$ \\
\hline Pitch suspension damping & $D_{\theta}$ & 31 & $\mathrm{kNm}(\mathrm{rad})^{-1}$ \\
\hline Roll suspension stiffness & $K_{\phi}$ & 178 & $\mathrm{kNm}(\mathrm{rad})^{-1}$ \\
\hline Roll suspension damping & $D_{\phi}$ & 16 & $\mathrm{kNm}(\mathrm{rad})^{-1}$ \\
\hline
\end{tabular}

Table 2.4: Wheel and tire-force model parameters used in the relations (2.18)-(2.29). The tire-force model parameters originate from (Pacejka, 2006), representing the behavior of a tire on dry asphalt.

(a) Wheel parameters.

\begin{tabular}{llll}
\hline Description & Notation & Value & Unit \\
\hline Rotational wheel inertia & $I_{w}$ & 2 & $\mathrm{kgm}^{2}$ \\
Wheel radius & $R_{w}$ & 0.3 & $\mathrm{~m}$ \\
Time constant of braking system & $\gamma_{T}$ & 0.1 & $\mathrm{~s}$ \\
Relaxation length & $\sigma$ & 0.3 & $\mathrm{~m}$ \\
\hline
\end{tabular}

(b) Longitudinal tire-force parameters.

(c) Lateral tire-force parameters.

\begin{tabular}{llllllll}
\cline { 1 - 2 } \cline { 5 - 7 } Notation & $i \in\{1,2\}$ & $i \in\{3,4\}$ & & Notation & $i \in\{1,2\}$ & $i \in\{3,4\}$ \\
\cline { 1 - 4 } \cline { 5 - 7 }$\mu_{x, i}$ & 1.20 & 1.20 & & & $\mu_{y, i}$ & 0.935 & 0.961 \\
$B_{x, i}$ & 11.7 & 11.1 & & $B_{y, i}$ & 8.86 & 9.30 \\
$C_{x, i}$ & 1.69 & 1.69 & & $C_{y, i}$ & 1.19 & 1.19 \\
$E_{x, i}$ & 0.378 & 0.362 & & $E_{y, i}$ & -1.21 & -1.11 \\
$B_{x 1, i}$ & 12.4 & 12.4 & & $B_{y 1, i}$ & 6.46 & 6.46 \\
$B_{x 2, i}$ & -10.8 & -10.8 & & $B_{y 2, i}$ & 4.20 & 4.20 \\
$C_{x \alpha, i}$ & 1.09 & 1.09 & & $C_{y \kappa, i}$ & 1.08 & 1.08 \\
\hline
\end{tabular}





\section{References}

K. Berntorp. Derivation of a six degrees-of-freedom ground-vehicle model for automotive applications. Technical Report 7627, Department of Automatic Control, Lund University, Sweden, 2013.

K. Berntorp. Particle Filtering and Optimal Control for Vehicles and Robots. PhD Theses TFRT-1101, Department of Automatic Control, Lund University, Sweden, 2014.

K. Berntorp, B. Olofsson, K. Lundahl, and L. Nielsen. Models and methodology for optimal trajectory generation in safety-critical road-vehicle manoeuvres. Vehicle System Dynamics, 52(10):1304-1332, 2014.

J. B. Cicchino. Effectiveness of forward collision warning and autonomous emergency braking systems in reducing front-to-rear crash rates. Accident Analysis \& Prevention, 99:142-152, 2017.

P. Cummings and D. C. Grossman. Antilock brakes and the risk of driver injury in a crash: A case-control study. Accident Analysis \& Prevention, 39(5): 995-1000, 2007.

G. Genta and L. Morello. The Automotive Chassis. Mechanical Engineering Series. Springer, London, United Kingdom, 2008.

T. Gordon and M. Lidberg. Automated driving and autonomous functions on road vehicles. Vehicle System Dynamics, 53(7):958-994, 2015.

C. J. Kahane and J. N. Dang. The long-term effect of ABS in passenger cars and LTVs. NHTSA Technical Report DOT HS 811 182, Evaluation Division, National Center for Statistics and Analysis, National Highway Traffic Safety Administration, U.S. Department of Transportation, August 2009.

N. R. Kapania and J. C. Gerdes. Design of a feedback-feedforward steering controller for accurate path tracking and stability at the limits of handling. Vehicle System Dynamics, 53(12):1687-1704, 2015. 
U. Kiencke and L. Nielsen. Automotive control systems: for engine, driveline and vehicle. Springer, Berlin, Germany, 2005.

K. Lundahl. Models and Critical Maneuvers for Road Vehicles. PhD thesis, Linköping Studies in Science and Technology. Dissertations 1763. Department of Electrical Engineering, Linköping University, Sweden, 2016.

K. Lundahl, J. Åslund, and L. Nielsen. Vehicle dynamics platform, experiments, and modeling aiming at critical maneuver handling. Technical Report 3064, Department of Electrical Engineering, Linköping University, Sweden, 2013.

A. Lyckegaard, T. Hels, and I. M. Bernhoft. Effectiveness of electronic stability control on single-vehicle accidents. Traffic Injury Prevention, 16(4):380-386, 2015.

D. Muoio. Ranked: The 18 companies most likely to get self-driving cars on the road first. Business Insider, 2017. URL http://nordic.businessinsider.com/ the-companies-most-likely-to-get-driverless-cars-on-the-road-first-2017-4. Accessed: 2018-04-04.

National Highway Traffic Safety Administration, U.S. Department of Transportation (NHTSA). Federal automated vehicles policy, 092016.

J. Ni and J. Hu. Dynamics control of autonomous vehicle at driving limits and experiment on an autonomous formula racing car. Mechanical Systems and Signal Processing, 90:154-174, 2017.

B. Olofsson. Topics in Machining with Industrial Robot Manipulators and Optimal Motion Control. PhD Theses TFRT-1108, Department of Automatic Control, Lund University, Sweden, 2015.

B. Olofsson, K. Lundahl, K. Berntorp, and L. Nielsen. An investigation of optimal vehicle maneuvers for different road conditions. In 7th IFAC Symposium on Advances in Automotive Control (AAC), Tokyo, Japan, 2013.

H. B. Pacejka. Tyre and Vehicle Dynamics. Butterworth-Heinemann, Oxford, United Kingdom, 2nd edition, 2006.

R. Rajamani. Vehicle Dynamics and Control. Springer, New York, United States, 2nd edition, 2012.

M. Rizzi, J. Strandroth, A. Kullgren, C. Tingvall, and B. Fildes. Effectiveness of motorcycle antilock braking systems (ABS) in reducing crashes, the first cross-national study. Traffic Injury Prevention, 16(2):177-183, 2015.

SAE International. Taxonomy and definitions for terms related to driving automation systems for on-road motor vehicles. Standard J3016_201609, 09 2016. 
R. S. Sharp and H. Peng. Vehicle dynamics applications of optimal control theory. Vehicle System Dynamics, 49(7):1073-1111, 2011.

C. Shelden. Prevention, the only cure for head injuries resulting from automobile accidents. Journal of the American Medical Association, 159(10):981-986, 1955.

Transportstyrelsen. Dödade, skadade, bilar i trafik, bensinleveranser och invånare samt dödade per 100000 bilar respektive invånare (årsvis fr.o.m. 1950), 2017. In Swedish.

H. Waschl, I. Kolmanovsky, M. Steinbuch, and L. del Re. Optimization and Optimal Control in Automotive Systems, volume 455 of Lecture Notes in Control and Information Sciences. Springer, Berlin, Germany, 2014.

J. Wong. Theory of Ground Vehicles. John Wiley \& Sons, New York, United States, 4th edition, 2008. 


\section{Papers}

The papers associated with this thesis have been removed for copyright reasons. For more details about these see:

http://urn.kb.se/resolve?urn=urn:nbn:se:liu:diva-147719 